\title{
Contagion Effect on Traditional Versus Innovative Products: Role of Consistency in "Essence" Transfer Process: An Abstract
}

\author{
Zhuofan Zhang
}

\begin{abstract}
This paper examines the contagion effect - perceived transfer of essence from the original manufacturing location - on traditional versus innovative products. Based on the concept of contagion and consistency, I introduce interference theory which states that consumers develop a sense of product essence by evaluating consistent product features over time. Accordingly, new products in product categories without much change are prone to a contagion effect in which consumers expect the essence to be retained. In contrast, product categories with great variability in product features over time are less likely to develop this essence. In these categories, the contagion effect is null, and consumers are receptive to radical and disruptive new product features. Thus the reception to totally radical innovation in a product category is explained by the contagion phenomenon.
\end{abstract}

References Available Upon Request.

Z. Zhang $(\bowtie)$

The University of Texas at El Paso, El Paso, TX, USA

e-mail: zzhang2@miners.utep.edu 